\title{
Onset and duration of effect of incobotulinumtoxinA, onabotulinumtoxinA, and abobotulinumtoxinA in the treatment of glabellar frown lines: a randomized, double-blind study
}

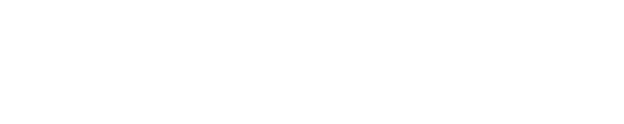

\author{
Thomas Rappl' \\ Daryousch Parvizi' \\ Herwig Friedl ${ }^{2}$ \\ Maria Wiedner' \\ Simone May' \\ Bettina Kranzelbinder ${ }^{3}$ \\ Paul Wurzer' \\ Bengt Hellbom ${ }^{1, \dagger}$ \\ 'Department of Plastic and \\ Reconstructive Surgery, Medical \\ University Graz, Graz, Austria; \\ ${ }^{2}$ Institute of Statistics, Graz University \\ of Technology, Graz, Austria; \\ ${ }^{3}$ Department of Dermatology, Medical \\ University Graz, Graz, Austria \\ †Professor Bengt Hellbom passed \\ away in September 2012
}

Correspondence: Thomas Rappl Department of Plastic and Reconstructive Surgery, Medical University Graz, Auenbruggerplatz 29, 8036 Graz, Austria

Tel +433330307

Fax +433330307

Email thomas.rappl@klinikum-graz.at
Background: Three botulinum neurotoxin type A preparations (incobotulinumtoxinA, onabotulinumtoxinA, and abobotulinumtoxinA) are widely approved in Europe and in the US for the treatment of glabellar frown lines. The purpose of this study was to determine and compare the time to onset and duration of treatment effect of incobotulinumtoxinA, onabotulinumtoxinA, and abobotulinumtoxinA for the treatment of glabellar frown lines.

Subjects and methods: Subjects aged 20-60 years with moderate to severe glabellar frown lines received one treatment of either 21 units $(U)$ incobotulinumtoxinA, $21 \mathrm{U}$ onabotulinumtoxinA, or $63 \mathrm{U}$ abobotulinumtoxinA. Assessments were made over a period of 180 days. Onset of treatment effect was defined as the day that the observer noted a decrease in glabellar muscle activity compared with baseline photographs and videos. Duration of treatment effect was defined as the time until glabellar muscle action returned to the baseline level. Analyses were performed using a Weibull $\log (\mathrm{T})$ regression model.

Results: The study enrolled 180 subjects; 60 per group. For all three products, onset of treatment effect occurred earlier in female subjects compared to male subjects. For both sexes, a significantly earlier time to onset of treatment effect was seen for incobotulinumtoxinA compared to onabotulinumtoxinA and abobotulinumtoxinA; in female subjects these times were 3.02 days, 5.29 days, and 5.32 days, respectively. The duration of treatment effect was longer for incobotulinumtoxinA compared to onabotulinumtoxinA and abobotulinumtoxinA; for all products, treatment effect duration was longer in females than in males. Time to onset was not a predictor of treatment duration.

Conclusion: IncobotulinumtoxinA demonstrated a more rapid onset and a longer duration of treatment effect than onabotulinumtoxinA (1:1 dose ratio) and abobotulinumtoxinA (1:3 dose ratio). Onset of effect was faster and duration of effect was longer in female subjects compared to male subjects.

Keywords: botulinum neurotoxin type A, glabellar frown lines, incobotulinumtoxinA, regression analysis

\section{Introduction}

The use of nonsurgical aesthetic techniques has become increasingly popular with both women and men seeking proven solutions to reverse the signs of aging. Of the nonsurgical techniques now available, those involving the injection of Clostridium botulinum neurotoxin type A (BoNT/A) have become among the most popular over the past few decades, and the use of such techniques increased by nearly $4000 \%$ in the US between 1997 and 2011. ${ }^{1}$ Vertical and horizontal hyperkinetic lines in the glabellar region of the 
face can give an erroneous impression of negative emotion ${ }^{2}$ and are the most common areas targeted by BoNT/A preparations, which are injected into the procerus and corrugator muscles to lessen their action. ${ }^{3}$ Currently, three BoNT/A preparations are approved widely in Europe and by the US Food and Drug Administration for the treatment of glabellar frown lines. IncobotulinumtoxinA (Xeomin ${ }^{\circledR} /$ Xeomeen $^{\circledR} /$ Bocouture $^{\circledR}$, XEOMIN Cosmetic ${ }^{\text {TM}}$; also known as NT 201, botulinum toxin type A [150 kDa]; Merz Pharmaceuticals $\mathrm{GmbH}$, Frankfurt am Main, Germany) is free from complexing proteins and in this respect differs from the other two BoNT/A preparations approved for this indication: onabotulinumtoxin $A\left(\right.$ Botox $^{\circledR} /$ Vistabel $^{\circledR} /$ Vistabex $^{\circledR}$; Allergan, Irvine, CA, USA) and abobotulinumtoxinA (Dysport ${ }^{\circledR}$; Medicis, Scottsdale, AZ, USA/Azzalure ${ }^{\circledR}$; Galderma UK, Watford, UK). Recent investigations have shown that complexing proteins have no therapeutic role and therefore represent an extra, redundant foreign protein load. ${ }^{4,5}$ IncobotulinumtoxinA is comprised of pure neurotoxin while other commercially available BoNT/A preparations, such as onabotulinumtoxinA and abobotulinumtoxinA, contain complexing proteins and are also likely to contain, in addition to the active neurotoxin, inactive or denatured neurotoxin/toxoid, resulting in a higher antigen load that increases the risk of neutralizing antibody formation $^{6}$ and may cause treatment failure. ${ }^{7-10}$ The proven efficacy and safety of incobotulinumtoxinA, onabotulinumtoxinA, and abobotulinumtoxinA for the treatment of glabellar frown lines has been demonstrated in several clinical trials in which all three treatments were associated with high response rates. ${ }^{11-17}$

Along with efficacy, time to onset and duration of effect are important factors that can influence subjects' level of satisfaction with their cosmetic treatments. Although numerous studies have investigated the efficacy of incobotulinumtoxinA, onabotulinumtoxinA, and abobotulinumtoxinA, only a few have focused specifically on their rapidity of onset of treatment effect. ${ }^{18-20}$ Onset of effect of BoNT/A treatment is often described as "relatively quick" or "rapid", ${ }^{12,14}$ but this parameter has not been assessed systematically on a daily basis following treatment; in most published trials, data were first obtained at variable time points, from 1 week postinjection, ${ }^{12}$ to as long as 2 weeks ${ }^{21-23}$ or even 4 weeks posttreatment. ${ }^{16,17}$ However, at these time points, the focus is often on efficacy rather than onset of effect. For incobotulinumtoxinA, results from randomized clinical trials have shown that improvement in the severity of glabellar frown lines is usually observed within 2 to 3 days after treatment, with maximal effect seen after 30 days. ${ }^{24}$ Prager et al recently confirmed these results in a study looking specifically at onset and duration of effect in which subjects were treated with incobotulinumtoxinA and assessed for 5 months. ${ }^{20}$ At maximum frown, high response rates of $95.2 \%$ (subjects with a $\geq 1$-point improvement compared with baseline on a validated 5-point scale) and $85.0 \%$ (subjects with a $\geq 2$-point improvement compared with baseline) were observed from 2 to 4 days after treatment. Overall, $84 \%$ of the maximal effect also occurred during this timeframe. ${ }^{20}$ Similarly, a median time to onset of effect of 3 days was reported in a prospective, randomized, double-blind Phase III trial investigating a single dose of incobotulinumtoxinA. ${ }^{25}$ An onset of effect described by the authors as occurring in the "first few days after treatment" has also been observed in a second, similarly designed, randomized Phase III trial, ${ }^{26}$ and in a long-term study assessing a maximum of eight treatment cycles of incobotulinumtoxinA over a 2-year period. ${ }^{27}$

Comparable times for onset of treatment effect have been reported for onabotulinumtoxinA and abobotulinumtoxinA. A study of onabotulinumtoxinA by Beer et al reported a rapid onset of effect (as assessed by subjects and physicians) in 45 women with moderate to severe glabellar frown lines; $77 \%$ of subjects and $87 \%$ of physicians reported onset of effect by day 2, but this subjective assessment of response was not correlated with facial wrinkle scale. ${ }^{18}$ In a subset analysis of four randomized, double-blind, placebo-controlled Phase III trials involving 1160 subjects receiving abobotulinumtoxinA for the treatment of glabellar frown lines of at least moderate severity at maximum frown, subjects reported median times to onset ranging from 2 to 4 days; however, investigator assessments were not performed. ${ }^{19}$

Duration of treatment effect is another parameter that is infrequently or inadequately investigated in clinical trials of BoNT/A preparations, with available evidence coming predominantly from single-agent, dose-ranging studies. ${ }^{14,15,21,22}$ In two dose-ranging studies investigating onabotulinumtoxinA in men and women with moderate to severe glabellar frown lines, longer duration of effect was associated with higher treatment doses $(20,30$, or $40 \mathrm{U}$ compared to $10 \mathrm{U}$ in women; 40, 60, or $80 \mathrm{U}$ compared to $20 \mathrm{U}$ in men). ${ }^{21,22}$ Moreover, the duration of treatment effect tended to be more durable at rest compared to maximum frown. ${ }^{21}$ Similarly, in a dose-ranging study of abobotulinumtoxinA ( $25 \mathrm{U}, 50$ $\mathrm{U}$, and $75 \mathrm{U}$ ) conducted by Ascher et al, durable responses in glabellar frown lines (as assessed by the investigator at rest) were observed in $64 \%-76 \%$ of subjects at 3 months and in approximately one-third of subjects up to 6 months. ${ }^{14}$ 
At maximum frown 3 months posttreatment, approximately $50 \%$ of subjects were responders (the effect of treatment persisted) with $50 \mathrm{U}$ and $75 \mathrm{U}$ doses but only $32.1 \%$ of subjects in the $25 \mathrm{U}$ group were responders at this time point. ${ }^{14}$ In a study comparing the efficacy of incobotulinumtoxinA with a $50 \%$ higher dose of onabotulinumtoxinA, half of a subset of 12 subjects followed up at 6 months posttreatment still showed at least a 1-point improvement compared with baseline on the Merz 5-point scale ${ }^{28}$ following treatment with either incobotulinumtoxinA (equivalent to $20 \mathrm{U}$ ) or onabotulinumtoxinA (equivalent to $30 \mathrm{U}$ ). ${ }^{29}$ Additionally, in the study by Prager et al, persistence of incobotulinumtoxinA treatment effect, defined as response of at least a 1-point improvement from baseline, was maintained in over $75 \%$ of subjects at 5 months postinjection. ${ }^{20}$

Ultimately, time to onset and duration of effect are important factors that have a considerable influence on subject satisfaction with cosmetic treatments, with implications for cost and convenience. In spite of this fact, few studies have compared the onset and duration of effect of incobotulinumtoxinA, onabotulinumtoxinA, and abobotulinumtoxinA. The aim of this study was to investigate whether these BoNT/A preparations display differences with regard to onset and duration of effect when used in a 1:1:3 dose conversion ratio. A further aim was to precisely determine for each product the time point during the first week after treatment at which the onset of effect occurred and how long the effect lasted.

\section{Materials and methods}

This was a double-blind, randomized, single-center study that took place between 2008 and 2011. The study was conducted according to the principles of the Declaration of Helsinki 1975, and consent was obtained from all participating subjects. Eligible male and female subjects met the following inclusion criteria: age 20-60 years, glabellar frown lines with a score of 1-3 (mild to severe) at maximum frown on the validated Merz 5-point scale (Figure 1), ${ }^{28}$ and either no prior BoNT/A treatment or previous treatment at least 1 year prior to the start of this study. Since the Merz 5-point scale was not available at the start of the study, photographs and videos for all subjects were reevaluated in 2012 using this scale for the analysis. Exclusion criteria included: presence of very severe frown lines, facial asymmetry, contraindicated medications (eg, anticoagulants), prior allergic reaction to BoNT/A treatment, prior injection into the glabellar area within the last year, infection or inflammation, pregnancy, and breastfeeding. Eligible subjects were assigned to groups of 60 and then randomized, using the randomizer program available at the Medical University of Graz (http:// www.randomizer.at), to receive treatment with either $21 \mathrm{U}$ incobotulinumtoxinA, $21 \mathrm{U}$ onabotulinumtoxinA, or 63 $\mathrm{U}$ abobotulinumtoxin $\mathrm{A}$; this represented a 1:1:3 dose ratio, which was the recommended conversion rate at the time of preparation of the study protocol. ${ }^{30}$ Vials of $100 \mathrm{U}$ of incobotulinumtoxin $\mathrm{A}$ and onabotulinumtoxinA were reconstituted as per the manufacturer's instructions, with the exception that the volume used was only $1.0 \mathrm{~mL}$. One $500 \mathrm{U}$ vial of abobotulinumtoxinA was reconstituted in $2.0 \mathrm{~mL}$. The injection volume was $0.21 \mathrm{~mL}$ for $21 \mathrm{U}$ incobotulinumtoxinA or onabotulinumtoxinA and $0.25 \mathrm{~mL}$ for $63 \mathrm{U}$ abobotulinumtoxinA. In order for the injecting physician to remain blinded to the product used, an assistant prepared the products for injection using special syringes (Figure 2) that did not have volume graduations but only had markings to show how much to inject per site. Therefore, the injection volumes were sufficiently similar regardless of treatment, to preserve the blinding of the injecting physician. Injection sites used are shown in Figure 3. Doses were chosen following previous investigations to identify the optimal number of units to inject with respect to muscle anatomy. The best results were achieved using $5 \mathrm{U}$ for incobotulinumtoxinA or onabotulinumtoxin $\mathrm{A}$ and $15 \mathrm{U}$ for abobotulinumtoxinA injected into the belly of the procerus

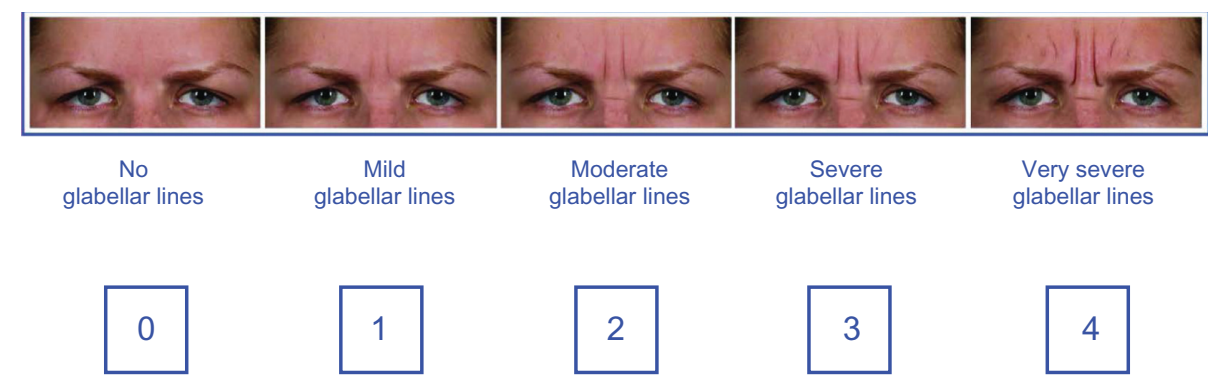

Figure I The validated Merz 5-point scale for glabellar frown lines at maximum frown.

Note: Reproduced with permission from Flynn TC, Carruthers A, Carruthers J, et al. Validated assessment scales for the upper face, Dermatologic Surgery, Wiley Periodicals, Inc. ${ }^{28}$ 


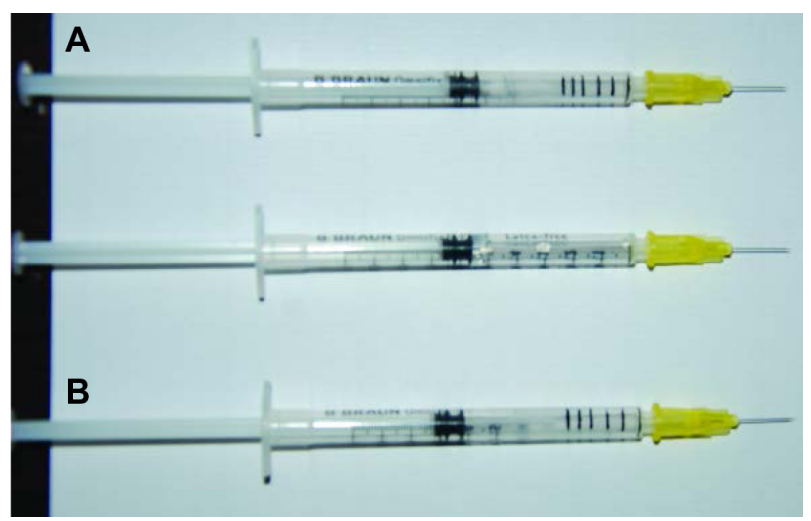

Figure 2 Syringes lacking numbered graduations used in order to maintain blinding of injecting physician.

Notes: (A) syringe containing onabotulinumtoxinA or incobotulinumtoxinA; (B) syringe containing abobotulinumtoxinA.

and corrugator supercilii muscles. These doses were also chosen as they appeared to result in a longer duration of effect in men.

Subjects attended 11 assessment visits to the clinic, on days $2,3,4,5,6,7,8,90,120,150$, and 180 . Standardized digital photographs and videos were taken at each visit at a focal distance of $40 \mathrm{~cm}$ (Nikon D800 camera, [Nikon Corporation, Tokyo, Japan]). The onset of treatment effect was defined as the day on which the observer (injecting physician) and one other physician noted a decrease of at least 1-point on the Merz 5-point scale compared with baseline (day 1). This was done by comparing the glabellar muscle activity of the subject at each visit with the video showing their baseline muscle activity prior to treatment. The duration of treatment effect was defined as the time until the observer first reported that the muscle action had returned to the same level as that seen at baseline (by comparison with the baseline video of glabellar muscle activity).

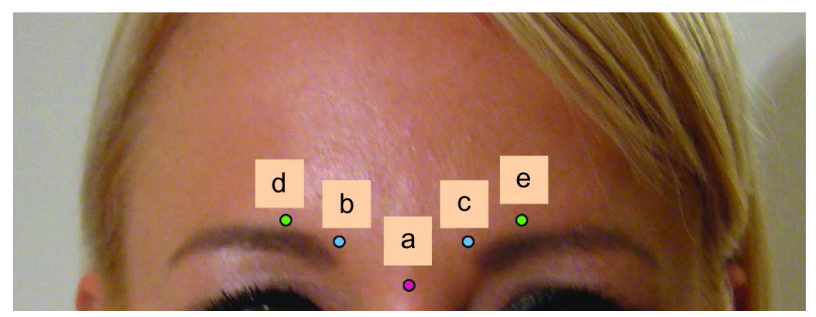

Figure 3 Injection sites.

Notes: (a) Procerus muscle; (b) right medial corrugator muscle; (c) left medial corrugator muscle; (d) right lateral corrugator muscle; (e) left lateral corrugator muscle. When subjects were injected with incobotulinumtoxinA or onabotulinumtoxinA, injection sites $a, b$, and c received $5 U$ each, while $d$ and e received $3 \mathrm{U}$ each. AbobotulinumtoxinA treatment consisted of $15 \mathrm{U}$ injections at sites $a, b$, and $c$, and $9 \cup$ at sites $d$ and $e$.

\section{Safety}

Safety assessments were conducted at each visit and any adverse events were recorded.

\section{Statistical analysis}

Analysis of the time to onset of treatment effect and recovery of muscle activity was mainly performed using statistical survival analysis methods. This included the simple regression model $\log (\mathrm{T})=\mathrm{x}^{\prime} \beta+\sigma \mathrm{Z}$, with the response variable $T$ describing the occurrence of the event of interest in days, $x$ ' $\beta$ being the linear predictor containing treatment and sex information, and $\mathrm{Z}$, a random variable from a specified distribution (eg, normal, lognormal, or Weibull distribution). To examine whether the time point of return to baseline glabellar muscle activity was dependent on time to onset, as before, a Weibull $\log (\mathrm{T})$ regression model was used. In addition, the time to onset information was allowed as a predictor of the time to recovery of glabellar muscle activity.

\section{Results}

Fifty-one female and nine male subjects were included in each of the three treatment groups, resulting in a total of 180 subjects. One female subject in the onabotulinumtoxinA group was excluded from the analysis due to failure to respond (in order to establish and compare the onset and duration of effect of the different products, it was essential that an effect was demonstrated). Subject demographics and baseline characteristics are shown in Table 1. All subjects had a normal body mass index (BMI $18.5-25 \mathrm{~kg} / \mathrm{m}^{2}$ ), were Caucasian, and had glabellar frown lines that were symmetrical. At baseline, a total of 26 subjects had a score of 1 on the validated Merz 5-point scale, 136 subjects had a score of 2 , and 18 subjects had a score of 3 .

\section{Onset of treatment effect (assessed by the injecting physician)}

Data for onset of treatment effect were available for 179 subjects. Under the assumption of a Weibull model for $\log (\mathrm{T})$, the median values for time to onset of treatment effect were estimated (Table 2). The hypothesis that all three treatments have the same median time to onset was rejected $(P<0.0001)$ and treatment identity was a significant primary predictor of time to onset. When subject sex was added to the model, a significant prediction was still obtained $(P=0.02)$, indicating that after allowing for the effect of the three different treatments, there was also the additional effect of sex on the median time to onset. Therefore, there was a significant difference between male and female subjects in time to onset 
Table I Subject demographics

\begin{tabular}{|c|c|c|c|c|}
\hline & $\begin{array}{l}\text { IncobotulinumtoxinA } \\
(n=60)\end{array}$ & $\begin{array}{l}\text { OnabotulinumtoxinA } \\
(n=59)\end{array}$ & $\begin{array}{l}\text { AbobotulinumtoxinA } \\
(n=60)\end{array}$ & $\begin{array}{l}\text { Total } \\
(n=179)\end{array}$ \\
\hline \multicolumn{5}{|l|}{ Sex } \\
\hline Male, n (\%) & $9(15)$ & $9(15)$ & $9(15)$ & $27(15)$ \\
\hline Female, n (\%) & $5 \mathrm{I}(85)$ & $50(85)$ & $51(85)$ & 152 (85) \\
\hline \multicolumn{5}{|l|}{ Age, years } \\
\hline Mean $( \pm S D)$ & $40.3(6.8)$ & $39.7(6.1)$ & $40.7(6.5)$ & $40.2(6.4)$ \\
\hline Range & $28-60$ & $31-60$ & $28-60$ & $28-60$ \\
\hline
\end{tabular}

Abbreviation: SD, standard deviation.

of effect, with female subjects experiencing an earlier time of onset compared to male subjects. In females, the median time to onset was 3.02 days for incobotulinumtoxinA, 5.29 days for onabotulinumtoxinA, and 5.32 days for abobotulinumtoxinA; the corresponding data for male subjects were 3.36, 5.89, and 5.93 days, respectively. KaplanMeier curves for time to onset of treatment effect in female and male subjects are shown in Figure 4. These curves were generated under the presumption that a treatment onset event had not yet occurred.

\section{Duration of treatment effect (assessed by the injecting physician)}

To aid comparison with the time to onset analysis, the time until glabellar muscle activity returned to the baseline level was also analyzed using a Weibull model. The calculated median values for return to baseline levels, based on subject sex and treatment, are shown in Table 3. When analyzing the data under a Weibull assumption, the hypothesis that both sexes have the same median duration was rejected $(P<0.0001)$, with sex being the primary predictor of treatment duration. The addition of treatment identity had only a small influence $(P=0.0735)$. Interestingly, this is the reverse of the situation for onset where treatment identity was the primary predictor for time to onset. Male subjects returned to the baseline glabellar muscle activity level at earlier time points (range, 115.81-121.14 days) than female subjects (range, 139.69-146.12 days). On the basis of median values, incobotulinumtoxinA appeared to have a longer duration of effect than onabotulinumtoxinA and abobotulinumtoxinA (Figure 5).
Interestingly, eight subjects (four from the incobotulinumtoxinA group and two each from the onabotulinumtoxin A and abobotulinumtoxinA groups) still showed an effect at day 180. All of these subjects were among the 26 subjects who were BoNT/A treatment naïve, and all had mild glabellar frown lines (with a score of 1 on the 5-point scale) at baseline.

\section{Dependence of duration of treatment effect on time to onset of effect}

A Weibull regression model for $\log (\mathrm{T})$ was used to investigate whether duration of effect was dependent on time to onset of effect. Time to onset information was allowed as a predictor in the model for recovery to baseline glabellar muscle activity levels. The analysis revealed that time to onset of treatment effect is not a predictor for the duration of effect.

\section{Safety}

Following treatment, only two subjects reported mild bruising, which resolved within 2 to 3 days. No serious adverse events were reported and there were no treatment-related discontinuations.

\section{Discussion}

This study was conducted to investigate whether there are differences in the onset and duration of effect of three approved BoNT/A preparations, incobotulinumtoxinA (21 U), onabotulinumtoxinA (21 U), and abobotulinumtoxinA (63 U), in the treatment of glabellar frown lines. Notably, the study aimed to pinpoint precisely, using an objective assessment

Table 2 Median onset of treatment effect

\begin{tabular}{llll}
\hline Sex & \multicolumn{2}{l}{ Median time to onset of treatment effect (days) } \\
\cline { 2 - 4 } & IncobotulinumtoxinA & OnabotulinumtoxinA & AbobotulinumtoxinA \\
\hline Female & 3.02 & 5.29 & 5.32 \\
Male & 3.36 & 5.89 & 5.93 \\
\hline
\end{tabular}



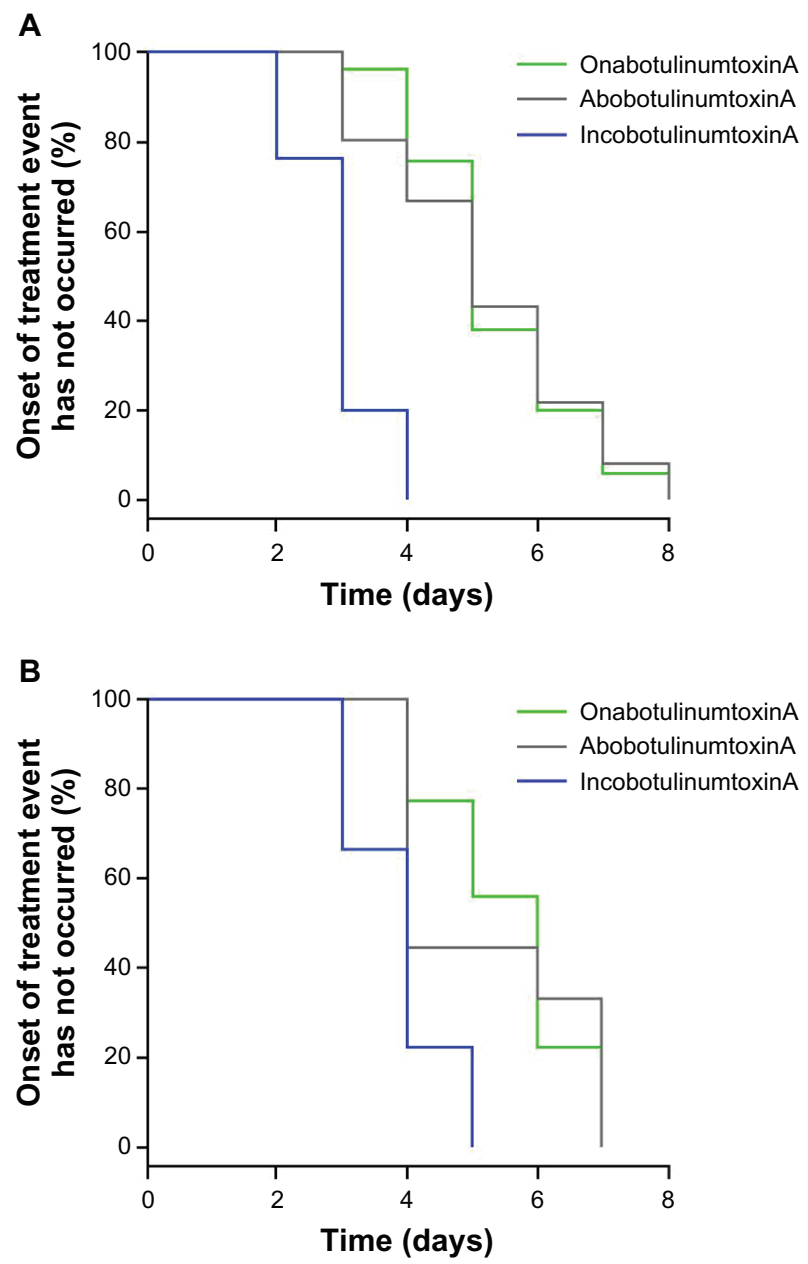

Figure 4 Kaplan-Meier curves for time to onset of treatment effect for (A) female and (B) male subjects.

method, the time point during the first week after treatment at which the onset of effect occurred for each product, and how long the effect lasted. This is important since, in the majority of BoNT/A studies, time to onset of effect has been reported solely by subjective patient self-assessments. Results from the present study showed that subjects treated with incobotulinumtoxinA had a significantly earlier onset of effect than those treated with either onabotulinumtoxinA or abobotulinumtoxinA. The faster onset of effect with incobotulinumtoxinA was seen despite the relative dose chosen for abobotulinumtoxinA being considerably higher than that recommended by its manufacturer (50 U for glabellar frown lines). The observed difference in time to onset of effect between the products may be due to a number of factors. The possibility that some BoNT/As contain denatured or inactive neurotoxin in addition to active neurotoxin ${ }^{6}$ may account for the slower onset of effect of this product as these components may compete with the active neurotoxin for binding sites on the surface of nerve terminals at the neuromuscular junction. This in turn might reduce the rate of internalization of active neurotoxin and delay the onset of action. It has been postulated that incobotulinumtoxinA contains active neurotoxin only ${ }^{6}$ and this might explain the more rapid onset of effect seen with incobotulinumtoxinA, as the active neurotoxin would not be competing with denatured or inactive neurotoxin.

With all three products, female subjects experienced a more rapid onset of effect than male subjects. This finding is unsurprising, as men have larger muscles and require higher doses than women ${ }^{21,22}$ in order to avoid an "underdose" situation in which the onset of effect occurs slightly later. The current study demonstrated that the duration of treatment effect was slightly longer for incobotulinumtoxinA compared to onabotulinumtoxinA or abobotulinumtoxinA, with female subjects experiencing a longer duration of effect than males. We have also demonstrated that there is no relationship between the time to onset of effect and the expected duration of effect.

For incobotulinumtoxinA, the time to onset of effect recorded in the current study is in line with results from a randomized, placebo-controlled Phase III trial, that reported onset of treatment effect at 3 days postinjection. ${ }^{25}$ The onset of effect times for onabotulinumtoxinA and abobotulinumtoxinA recorded in this study were slightly longer than those reported in previous studies; however, there are possible explanations for these differences. For instance, in an earlier study that reported a more rapid onset of effect for onabotulinumtoxinA, $48 \%$ of subjects noted treatment onset by day $1 .{ }^{18}$ However, this rating was obtained in response to the question, "Since being injected have you noticed any effect on the appearance of your frown lines (lines between the eyebrows)?" and was not correlated with a facial wrinkle rating scale. ${ }^{18}$ Therefore, some ambiguity may exist with regard to the exact definition of treatment onset according to subjects. In addition, the current study used a different indicator of

Table 3 Median duration of treatment effect

\begin{tabular}{llll}
\hline Sex & \multicolumn{2}{l}{ Median time for duration of treatment effect (days) } & AbobotulinumtoxinA \\
\cline { 2 - 4 } & IncobotulinumtoxinA & OnabotulinumtoxinA & 139.69 \\
\hline Female & 146.12 & 140.65 & 115.81 \\
Male & 121.14 & 116.61 & 1 \\
\hline
\end{tabular}



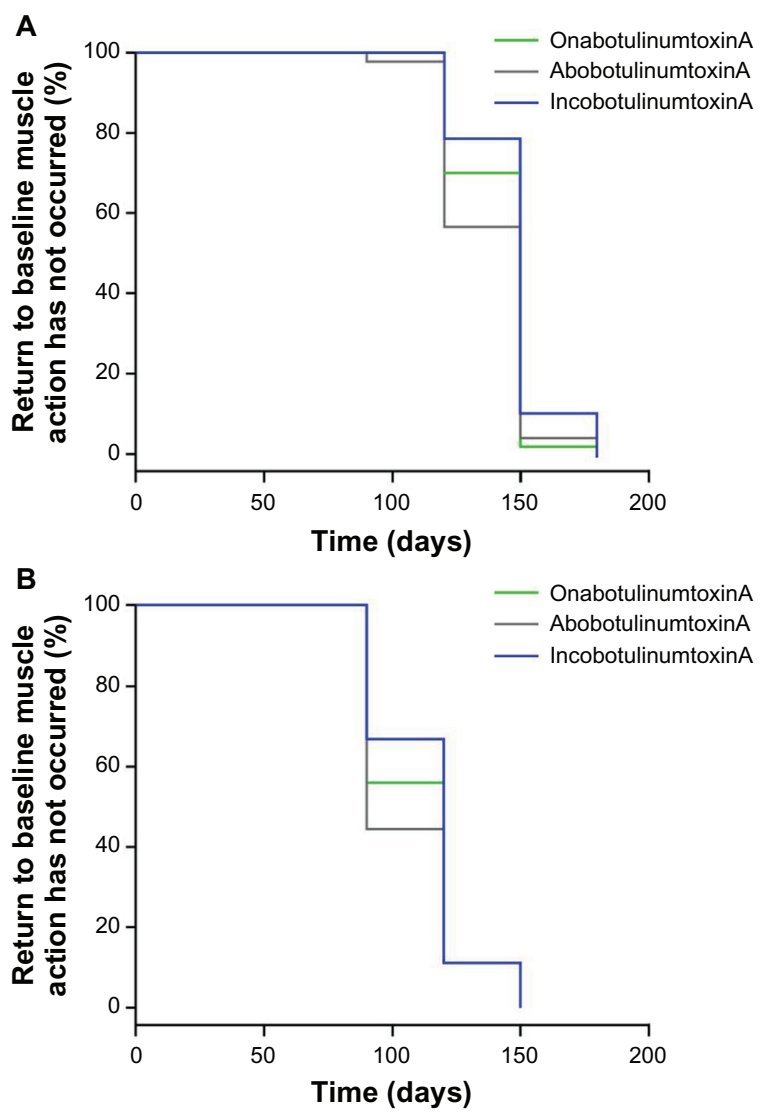

Figure $\mathbf{5}$ Kaplan-Meier curves for duration of treatment effect for (A) female and (B) male subjects.

onset, a decrease in muscle activity compared with baseline and in this study onset was observer assessed and not subject assessed. For abobotulinumtoxinA, a median time of onset of 2-4 days was reported by Schlessinger et al, but these results were obtained from patient self-assessment diaries that were not substantiated by investigator assessment. ${ }^{19}$ Additionally, the dose conversion ratio of 1:3 for abobotulinumtoxinA that was chosen in the current study was based on that used in older studies in therapeutic indications, ${ }^{30}$ and was in the middle of the range of relative strength of action for cosmetic indications. ${ }^{31}$ The abobotulinumtoxinA dose of $63 \mathrm{U}$ was higher than the $50 \mathrm{U}$ dose currently recommended for glabellar frown lines. ${ }^{32}$ Indeed, dose conversion ratios of less than 1:3 (such as 1:2.5) are considered to be more appropriate now. ${ }^{33}$ The use of a higher concentration in this study may affect the outcome. Differences in study population and the methods of measuring onset of treatment effect between trials mean that caution should be applied when comparing the results of different studies.

Regarding duration of effect, it is also difficult to compare the results from the present study with those from earlier dose ranging studies. Herein, we used the mean number of days before relapse to determine treatment duration, whereas in previous studies, duration was measured using the percentages of responders at specific time points. ${ }^{14,15}$ In studies that reported duration of effect conservatively (as a return to baseline on two consecutive visits approximately 30 days apart), the duration of effect of BoNT/A was 3-6 months or longer for women, ${ }^{21}$ at least 3-4 months for most men, and up to 6 months in some. ${ }^{22}$ This finding is similar to that reported in the present study and suggests that the effect of treatment may last longer in women; however, it is important to bear in mind that these dose ranging studies used higher doses in men compared with women, and that a dose dependent effect on duration was observed in men. We did not find a correlation between strength of response after day 30 and duration of effect.

It is interesting to note that almost one-third of the treatment-naïve subjects ( 8 of 26 subjects) still showed a response at day 180 . These eight subjects also had mild wrinkles at baseline and very thin corrugator muscles; it is possible that these factors contributed to the longevity of the response in these subjects.

A limitation of the current study is that observations to assess treatment duration were only performed at four individual time points $(90,120,150$, and 180 days after treatment) and censored information was not available for time periods between those four study visits; therefore, the results for duration of effect need to be interpreted with caution. Differences in study design may account for the differences in median time to onset and duration of treatment effect reported in this and other studies, emphasizing the importance of a large study that directly compares these parameters. To the authors' knowledge, this is the first large study comparing the onset of treatment effect between incobotulinumtoxin $\mathrm{A}$, onabotulinumtoxin $\mathrm{A}$, and abobotulinumtoxinA by physician assessment daily for 7 days following injection.

\section{Conclusion}

The results of this study indicate that incobotulinumtoxinA, at the minimal dose of $21 \mathrm{U}$, has a faster onset of effect than either onabotulinumtoxinA ( $21 \mathrm{U}$ ) or abobotulinumtoxinA (63 U) for treatment of glabellar frown lines. For all three BoNT/A products, female subjects experienced a more rapid onset of effect than male subjects, and also a slightly longer duration of treatment effect, when a similar dose is applied. 


\section{Acknowledgments}

This publication is dedicated to the memory of Prof Bengt Hellbom, who was involved with this work and sadly passed away in September 2012. Editorial assistance was provided by Ogilvy 4D, Oxford, UK and this was funded by Merz Pharmaceuticals GmbH, Frankfurt am Main, Germany.

\section{Disclosure}

Thomas Rappl has conducted speaker activities for Merz, Croma Pharma, MD-Skin Solutions, Johnson \& Johnson, and Smith \& Nephew. The authors report no other conflicts of interest in this work.

\section{References}

1. The American Society for Aesthetic Plastic Surgery statistics. Available from: http://www.surgery.org/sites/default/files/ASAPS-2011-Stats.pdf. Accessed April 4, 2012.

2. Cox SE, Finn JC. Social implications of hyperdynamic facial lines and patient satisfaction outcomes. Int Ophthalmol Clin. 2005;45(3): $13-24$.

3. Fedok FG. Advances in minimally invasive facial rejuvenation. Curr Opin Otolaryngol Head Neck Surg. 2008;16(4):359-368.

4. Eisele KH, Fink K, Vey M, Taylor HV. Studies on the dissociation of botulinum neurotoxin type A complexes. Toxicon. 2011;57(4):555-565.

5. Frevert J, Dressler D. Complexing proteins in botulinum toxin type A drugs: a help or a hindrance? Biologics. 2010;4:325-332.

6. Frevert J. Content of botulinum neurotoxin in Botox $^{\mathbb{\circledR}} /$ Vistabel $^{\mathbb{\Phi}}$, Dysport $^{\mathbb{1} /}$ Azzalure $^{\circledR}$, and Xeomin ${ }^{\circledR} /$ Bocouture $^{\circledR}$. Drugs R D. 2010;10(2):67-73.

7. Dressler D, Adib Saberi F. New formulation of Botox: complete antibody-induced treatment failure in cervical dystonia. $J$ Neurol Neurosurg Psychiatry. 2007;78(1):108-109.

8. Dressler D, Wohlfahrt K, Meyer-Rogge E, Wiest L, Bigalke H. Antibodyinduced failure of botulinum toxin a therapy in cosmetic indications. Dermatol Surg. 2010;36(Suppl 4):2182-2187.

9. Jankovic J, Vuong KD, Ahsan J. Comparison of efficacy and immunogenicity of original versus current botulinum toxin in cervical dystonia. Neurology. 2003;60(7):1186-1188.

10. Stengel G, Bee EK. Antibody-induced secondary treatment failure in a patient treated with botulinum toxin type A for glabellar frown lines. Clin Interv Aging. 2011;6:281-284.

11. Carruthers A, Carruthers J, Lowe NJ, et al. One-year, randomised, multicenter, two-period study of the safety and efficacy of repeated treatments with botulinum toxin type A in patients with glabellar lines. J Clin Res. 2004;7:1-20.

12. Carruthers JA, Lowe NJ, Menter MA, et al. A multicenter, doubleblind, randomized, placebo-controlled study of the efficacy and safety of botulinum toxin type A in the treatment of glabellar lines. JAm Acad Dermatol. 2002;46(6):840-849.

13. Carruthers JD, Lowe NJ, Menter MA, Gibson J, Eadie N; Botox Glabellar Lines II Study Group. Double-blind, placebo-controlled study of the safety and efficacy of botulinum toxin type A for patients with glabellar lines. Plast Reconstr Surg. 2003;112(4):1089-1098.

14. Ascher B, Zakine B, Kestemont P, Baspeyras M, Bougara A, Santini J. A multicenter, randomized, double-blind, placebo-controlled study of efficacy and safety of 3 doses of botulinum toxin A in the treatment of glabellar lines. J Am Acad Dermatol. 2004;51(2):223-233.

15. Monheit G, Carruthers A, Brandt F, Rand R. A randomized, double-blind, placebo-controlled study of botulinum toxin type A for the treatment of glabellar lines: determination of optimal dose. Dermatol Surg. 2007;33(1 Spec No):S51-S59.
16. Imhof M, Kühne U. A Phase III study of incobotulinumtoxinA in the treatment of glabellar frown lines. J Clin Aesthet Dermatol. 2011;4(10): 28-34.

17. Sattler G, Callander M, Grablowitz D, et al. Noninferiority of incobotulinumtoxinA, free from complexing proteins, compared with another botulinum toxin type A in the treatment of glabellar frown lines. Dermatol Surg. 2010;36(Suppl 4):2146-2154.

18. Beer KR, Boyd C, Patel RK, Bowen B, James SP, Brin MF. Rapid onset of response and patient-reported outcomes after onabotulinumtoxinA treatment of moderate-to-severe glabellar lines. J Drugs Dermatol. 2011;10(1):39-44.

19. Schlessinger J, Monheit G, Kane MA, Mendelsohn N. Time to onset of response of abobotulinumtoxinA in the treatment of glabellar lines: a subset analysis of phase 3 clinical trials of a new botulinum toxin type A. Dermatol Surg. 2011;37(10):1434-1442.

20. Prager W, Bee EK, Havermann I, Zschocke I. Onset, longevity and subject satisfaction with incobotulinumtoxinA for the treatment of glabellar frown lines: a single-arm, prospective clinical study. Clin Interv Aging. In press 2013.

21. Carruthers A, Carruthers J, Said S. Dose-ranging study of botulinum toxin type A in the treatment of glabellar rhytids in females. Dermatol Surg. 2005;31(4):414-422.

22. Carruthers A, Carruthers J. Prospective, double-blind, randomized, parallel-group, dose-ranging study of botulinum toxin type A in men with glabellar rhytids. Dermatol Surg. 2005;31(10):1297-1303.

23. Blitzer A, Binder WJ, Aviv JE, Keen MS, Brin MF. The management of hyperfunctional facial lines with botulinum toxin. A collaborative study of 210 injection sites in 162 patients. Arch Otolaryngol Head Neck Surg. 1997;123(4):389-392.

24. Bocouture ${ }^{\circledR}$ Summary of Product Characteristics. Merz Pharmaceuticals GmbH. 2013. Available from: http://www.medicines.org.uk/emc/ medicine/23251. Accessed February 10, 2013.

25. Hanke CW, Narins RS, Brandt F, et al. A randomized, placebo-controlled, double-blind Phase III trial investigating the efficacy and safety of incobotulinumtoxinA in the treatment of glabellar frown lines using a stringent composite endpoint. Dermatol Surg. In press 2013.

26. Carruthers A, Carruthers J, Coleman WP 3rd, et al. Multicenter, randomized, Phase III study of a single dose of incobotulinumtoxinA, free from complexing proteins, in the treatment of glabellar frown lines. Dermatol Surg. Epub February 4, 2013.

27. Rzany B, Flynn T, Schlöbe A, Heinz M, Harrington L. Long-term results for incobotulinumtoxinA in the treatment of glabellar frown lines. Dermatol Surg. 2013;39(1 Pt 1):95-103.

28. Flynn TC, Carruthers A, Carruthers J, et al. Validated assessment scales for the upper face. Dermatol Surg. 2012;38(2 Spec No):309-319.

29. Prager W, Rappl T. Phase IV study comparing incobotulinumtoxinA and onabotulinumtoxinA using a 1:1.5 dose-conversion ratio for the treatment of glabellar frown lines. J Cosmet Dermatol. 2012; 11(4):267-271.

30. Ranoux D, Gury C, Fondarai J, Mas JL, Zuber M. Respective potencies of Botox and Dysport: a double blind, randomised, crossover study in cervical dystonia. J Neurol Neurosurg Psychiatry. 2002;72(4): 459-462.

31. Carruthers A, Carruthers J. Botulinum toxin products overview. Skin Therapy Lett. 2008;13(6):1-4.

32. Azzalure ${ }^{\circledR}$ Summary of Product Characteristics. Galderma (UK) Ltd. 2013. Available from: http://www.medicines.org.uk/emc/medicine/21985. Accessed February 13, 2013.

33. Karsai S, Raulin C. Current evidence on the unit equivalence of different botulinum neurotoxin A formulations and recommendations for clinical practice in dermatology. Dermatol Surg. 2009;35(1):1-8. 


\section{Publish your work in this journal}

Clinical, Cosmetic and Investigational Dermatology is an international, peer-reviewed, open access, online journal that focuses on the latest clinical and experimental research in all aspects of skin disease and cosmetic interventions. All areas of dermatology will be covered; contributions will be welcomed from all clinicians and basic science researchers globally. This journal is indexed on CAS. The manuscript management system is completely online and includes a very quick and fair peer-review system, which is all easy to use. Visit http://www.dovepress.com/testimonials.php to read real quotes from published authors.

Submit your manuscript here: http://www.dovepress.com/clinical-cosmetic-and-investigational-dermatology-journal 